Musées, Patrimoine et Culture scientifiques et techniques

$186 \mid 2019$

novembre-décembre 2019

\title{
Accueillir les publics autistes au musée
}

Cyrielle Leriche

\section{OpenEdition \\ Journals}

Édition électronique

URL : http://journals.openedition.org/ocim/3470

DOI : $10.4000 /$ ocim.3470

ISSN : 2108-646X

Éditeur

OCIM

Édition imprimée

Date de publication : 1 novembre 2019

Pagination : $44-51$

ISSN : 0994-1908

Référence électronique

Cyrielle Leriche, "Accueillir les publics autistes au musée », La Lettre de l'OCIM [En ligne], 186 | 2019, mis en ligne le 01 novembre 2020, consulté le 24 janvier 2021. URL : http://journals.openedition.org/ ocim/3470; DOI : https://doi.org/10.4000/ocim.3470

Ce document a été généré automatiquement le 24 janvier 2021.

Tous droits réservés 


\section{Accueillir les publics autistes au musée}

\section{Cyrielle Leriche}

La salle des antiquités grecques préclassiques au musée du Louvre.

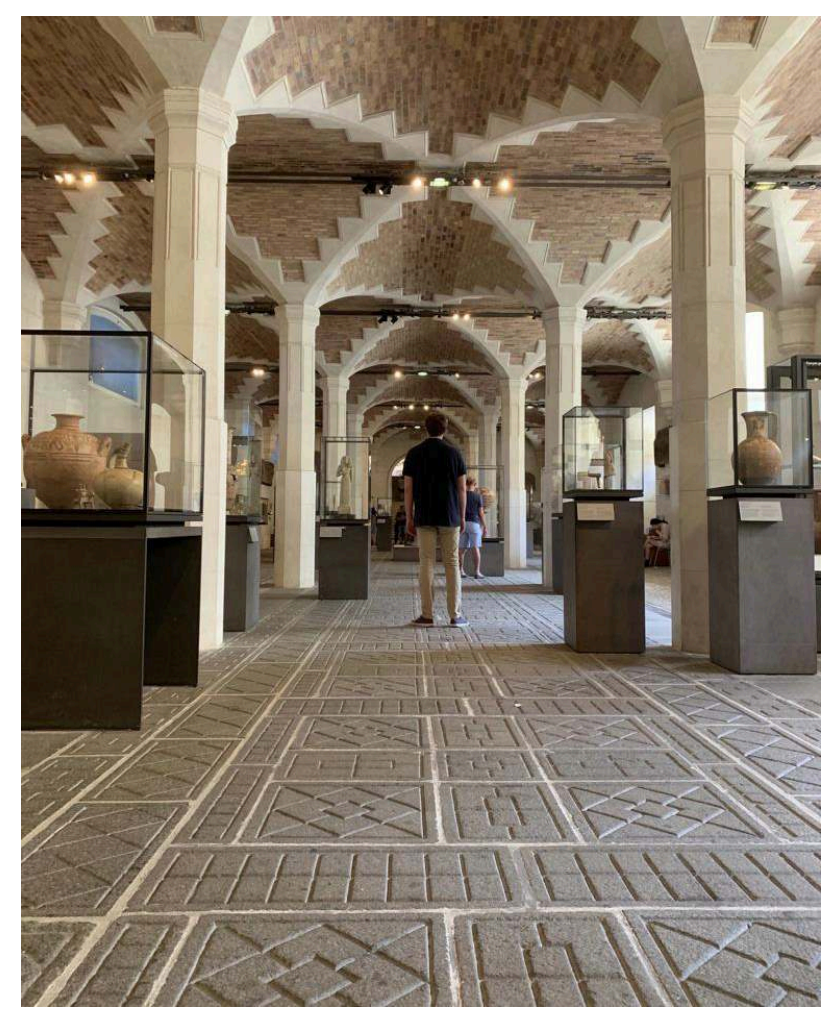

(C) Cyrielle Leriche

Depuis la loi du 11 février $2005^{1}$, la politique muséale tend à ouvrir ses portes aux publics dits «spécifiques ». Toutefois, bien que ces efforts soient notables, certaines 
formes d'handicaps sont sous-représentées parmi le public des musées : c'est notamment le cas de l'autisme.

2 Les visiteurs présentant des troubles du spectre autistique (TSA) sont encore marginaux dans les institutions culturelles. Cet état de fait se lit sous plusieurs axes. Par la nature opposée de ces deux mondes d'abord : l'autisme se caractérise par un repli sur soi avec des intérêts intellectuels restreints tandis que le musée prône une ouverture sociale et culturelle. Mais aussi de par les idées reçues sur cette pathologie qui leur ferment parfois les portes de la culture en questionnant la légitimité de leur venue.

3 Il semble pourtant qu'un lieu rempli d'histoire, d'art et surtout d'images, soit un lieu rêvé pour les visiteurs avec TSA qui ont une pensée en images (et non conceptuelle ou langagière comme c'est le cas pour beaucoup de neurotypiques²). C'est aussi un lieu fabuleux de découvertes et de pratiques sociales.

Les institutions muséales françaises ouvrent progressivement leurs portes aux visiteurs avec autisme. Le travail de recherche effectué ${ }^{3}$ 'inscrit donc dans un axe novateur qui tend à comprendre le handicap pour proposer une prise en charge adaptée aux personnes avec TSA et montre grâce à des exemples de musées que ce qui semblait a priori et peut-être inconciliable n'était en réalité pas impossible. Sur ces résultats encourageants, une étude expérimentale ${ }^{4}$ a été menée dans les salles du musée du Louvre pendant 6 mois. Elle tend à montrer que les troubles sensoriels constituent un frein majeur à la réception du discours artistique par un public avec un autisme, sans retard mental ni déficit langagier.

\section{Les Troubles du Spectre Autistique (TSA)}

Les TSA sont des troubles neurodéveloppementaux (apparition durant le développement de l'enfant) qui se caractérisent par la « dyade autistique ». Ce vocable regroupe les deux conditions primordiales du diagnostic de l'autisme : troubles de la communication (difficultés ou impossibilités à verbaliser) et troubles sociaux (problèmes de compréhension des codes de la société) ; centres d'intérêts restreints et répétitifs (faire tourner une roue indéfiniment...).

Cependant, l'autisme ne peut être réduit uniquement à cette dyade. L'appellation de « troubles du spectre autistique » a d'ailleurs été choisie pour englober au maximum la très grande variété des troubles liés à l'autisme : allant d'un QI moyen à une déficience intellectuelle en passant par un haut quotient intellectuel ${ }^{5}$, les TSA s'accompagnent généralement de troubles de la motricité, du langage, du comportement, de l'attention... Ces troubles associés sont propres à chaque individu et ne suivent en aucun cas un schéma général. 


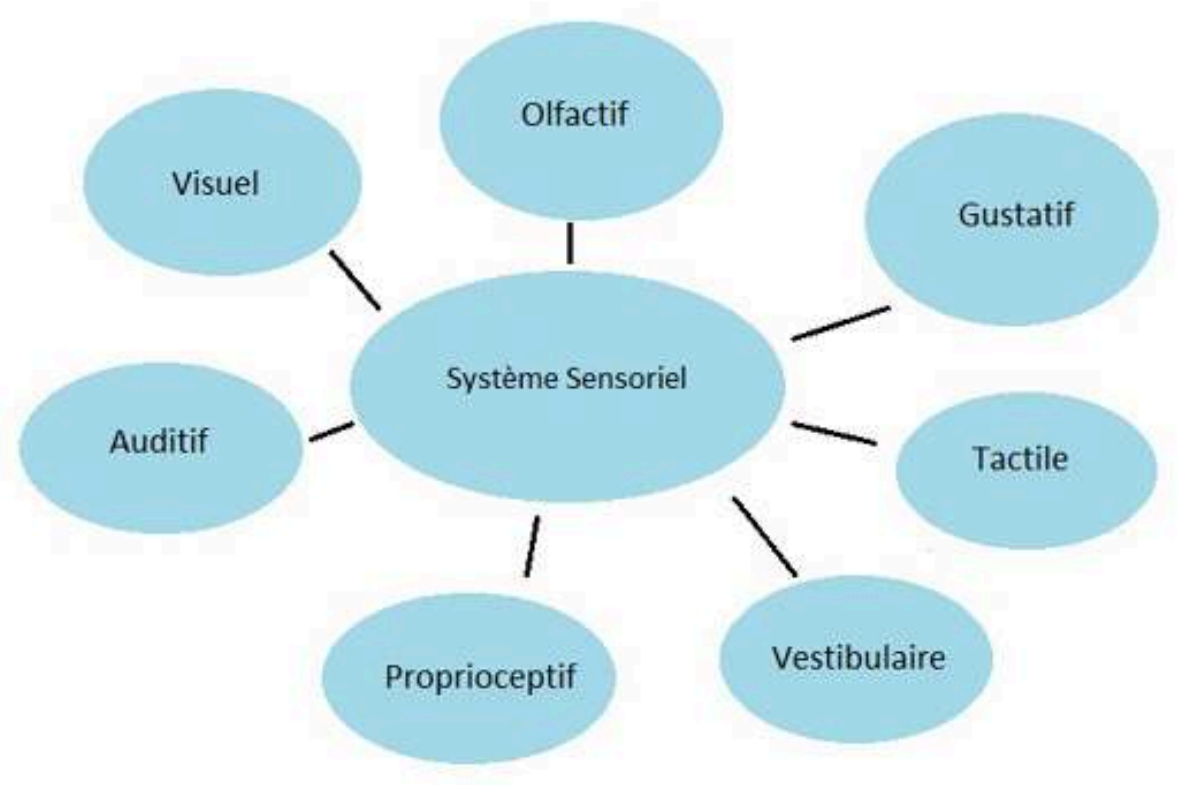

(c) Schéma du système sensoriel (http://aspieconseil.com)

Dans les TSA, les troubles sensoriels sont récurrents. Ils varient en forme et en intensité selon les individus et affectent un ou plusieurs sens (visuel, auditif, olfactif, tactile, gustatif) au travers de plusieurs organes sensoriels (yeux, oreilles, nez, langue). Ces troubles peuvent se traduire par une hypersensibilité aux stimuli sensoriels, avec une réception beaucoup plus intense du stimulus, ou à l'inverse une hyposensibilité. Selon l'hyper ou l'hyposensibilité, les personnes ayant des particularités sensorielles peuvent avoir du mal à gérer et traiter l'information sensorielle au quotidien. Cela peut conduire à de l'anxiété, de la colère et même à une véritable douleur physique. Ainsi, le grincement d'une porte peut engendrer une douleur auditive sévère dans le cas d'une hypersensibilité auditive ou au contraire être recherché par la personne comme une addiction pour une hyposensibilité.

Enfin, certaines particularités de l'autisme sont également importantes à prendre en compte dans une recherche portant sur ces publics dans les musées. Premièrement, la pensée en image qui rejoint les troubles sensoriels visuels. Les personnes avec TSA perçoivent le langage et les concepts associés sous forme d'images. À l'évocation du mot « porte » c'est une mini-vidéo de toutes les portes qu'ils ont pu voir dans leurs vies qu'ils entrevoient et non le concept langagier de " porte ». Une deuxième particularité est celle de la pensée en détails poussant un individu avec TSA à percevoir une personne ou un objet dans ses détails et non dans sa globalité. La vision périphérique est également très développée chez les personnes avec autisme. Surplombant la vision focale, elle les pousse à mieux voir ce qui se situe autour d'eux et par conséquent à les déconcentrer. Enfin, la peur de l'échec et l'imprévu peuvent être à l'origine d'angoisses les conduisant à abandonner ou renoncer à un projet.

7 Ces troubles, plus ou moins exacerbés, constituent le spectre de l'autisme et c'est pourquoi chaque forme d'autisme est spécifique. Cela rend ainsi impossible un schéma 
universel pour la prise en charge de l'autisme et par conséquent la création d'un programme muséal «type ».

\section{L'autisme et les muséesau niveau international}

8 À un niveau international, trois éléments semblent facteurs d'inclusion pour les publics avec TSA dans les musées : les dispositifs d'aides à la visite, le bien-être et la visée thérapeutique.

9 Comme le Metropolitan Museum de New York ou le musée du Louvre de Paris, certains musées mettent à disposition sur leurs sites internet des aides à la visite ${ }^{6}$. Ces fichiers permettent aux visiteurs, désireux de se rendre spontanément au musée et non en visite guidée, de construire leurs parcours de visite à l'avance. Ainsi, les visiteurs peuvent se renseigner sur l'itinéraire à suivre pour se rendre au musée, l'entrée dans le musée, les parcours de visite conseillés ou encore le règlement interne du musée. Ces fichiers sont élaborés à l'aide d'outils visuels, la pensée en image impliquant que les informations soient présentées visuellement à l'aide de pictogrammes ou d'images.

Une fois la visite préparée en amont, ces aides à la visite peuvent prendre une toute autre forme in situ comme au Victoria \& Albert Museum de Londres qui propose aux visiteurs des « sacs sensoriels » incluant des cartes du musée, des guides, des livretsjeux pour animer la visite, mais également des protège-oreilles pour diminuer le bruit ambiant $^{7}$. Ces aides à la visite sont un outil primordial permettant d'anticiper et préparer sa venue en palliant les imprévus - et donc les potentielles angoisses qu'ils engendrent -, mais permettent aussi de rendre la visite attractive, ludique et confortable.

\section{Emmanuel Alescio, participant au programme L'art dans tous ses étatsau MBAM.}

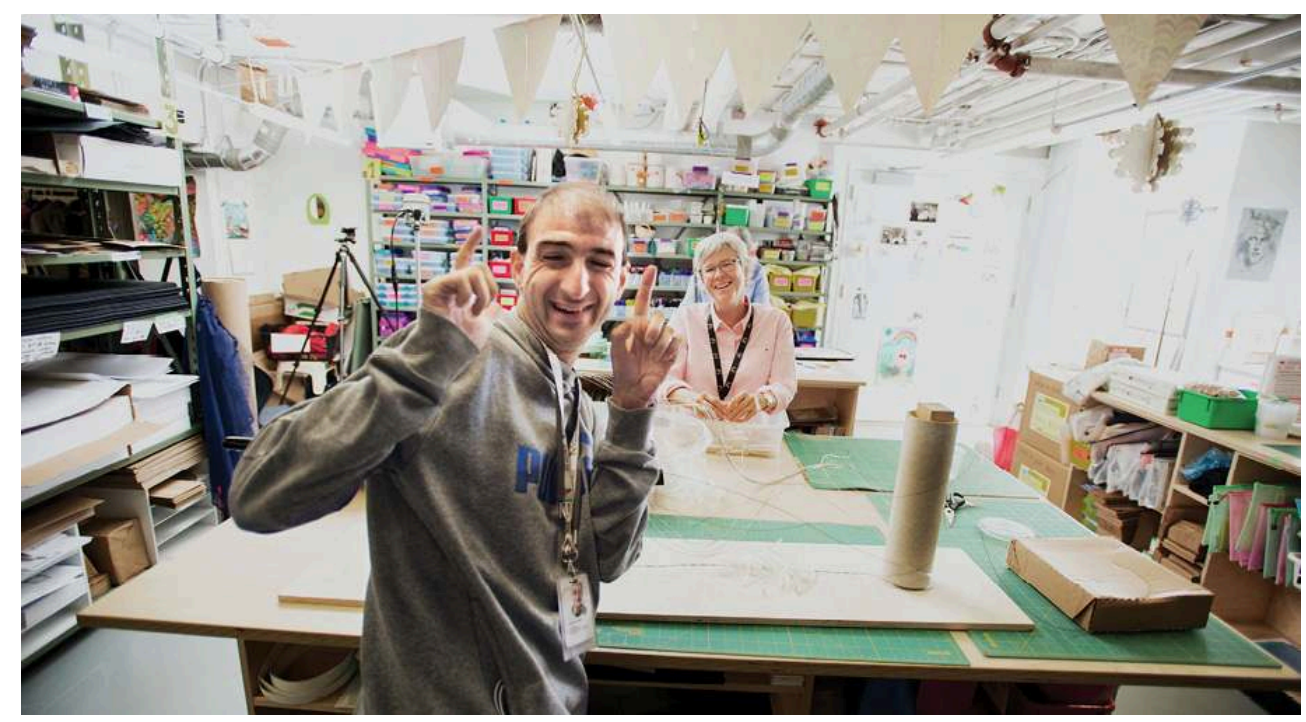

(c) Jean-François Brière

11 Le bien-être est un axe majeur et primordial pour nombre de musées. L'exemple du Children's Museum of New Hampshire, à Dover, est ici intéressant car il permet aux familles de passer un agréable moment au sein de son institution dans un environnement sécurisé et serein ${ }^{8}$. Les familles sont conviées deux heures avant 
l'ouverture du musée pour que les enfants puissent y prendre leurs marques à l'abri de la foule et sont vivement invitées à rester une fois le public présent. Ici, le programme mise sur le bien-être des participants pour les familiariser avec le lieu et les inciter à rester, à revenir.

Enfin, rares sont les musées ayant une visée thérapeutique au sein de leurs programmes. C'est pourtant cette dernière thématique qui a été retenue. Le musée des Beaux-Arts de Montréal (MBAM) en est une figure majeure grâce à son programme L'art dans tous ses états où l'art se transforme en moyen alternatif pour connaître l'autisme et offrir à travers le musée un lieu d'épanouissement et d'apprentissage des schémas sociaux ${ }^{9}$. Ce programme permet aux participants d'utiliser les collections du MBAM pour approfondir leurs connaissances des émotions et des comportements sociaux à travers les scènes représentées dans les œuvres.

Ces trois champs couvrent trois pans bien différents de la prise en charge pour accueillir les personnes atteintes d'autisme au musée. On constate ainsi que les TSA et le monde muséal ne sont en rien incompatibles : la compréhension des troubles, liée à l'adaptation muséale, pouvant conduire à une visite de ce public dans les institutions culturelles.

\section{L'approche expérimentale}

L'étude menée avait pour but :

- d'observer si l'environnement sensoriel du musée (c'est-à-dire tout ce qui a trait à la sensorialité du visiteur avec TSA dans le musée) est un obstacle à la réception et à la compréhension d'un discours de médiation pour un public présentant un trouble du spectre autistique sans retard mental ni déficit langagier ;

16 - de déterminer si la réception des stimuli sensoriels en musée varie selon l'âge des participants.

\section{Les fondements de l'étude :échantillon et outils d'analyses}

17 L'échantillon regroupait dix personnes " adolescentes » entre 10 et 19 ans et 10 personnes « adultes » entre 20 et 50 ans, sans retard mental ni déficience langagière. Le fait que tous les participants aient le même niveau était déterminant pour avoir une base d'étude solide et homogène. Le choix de recruter des autistes dits « légers » a été motivé par leurs capacités verbales leur permettant d'exprimer plus facilement leurs vécus vis-à-vis de la visite et de répondre aux questionnaires.

L'évaluation s'est faite en trois étapes. Tout d'abord, il a été question de mesurer le degré de gêne qu'engendre la sensorialité au quotidien. Cette mesure permettait par la suite d'avoir une référence pour comparer la gêne induite par la sensorialité au quotidien à celle induite à l'instant $\mathrm{T}$ de la visite. Pour se faire un questionnaire sensoriel tiré du Profil sensoriel de Winnie Dunn était soumis au participant avant la visite. Ce questionnaire permet de savoir si les participants se situent dans une " performance typique» (niveau de gêne sensorielle " normale »), une " différence probable » (niveau de gêne sensorielle marqué) ou une « différence avérée » (niveau de gêne sensorielle très important). 
19 À la fin de la visite, deux outils étaient présentés aux participants : un premier questionnaire, fermé, permettant d'évaluer la compréhension de la visite et une échelle colorée allant de " pas du tout gêné » à " extrêmement gêné », permettant de mesurer le vécu sensoriel en musée des participants et d'identifier ce qui les avait gênés - ou non - durant la visite (vitrine, bruit...).

Liste des éléments sensoriels (en haut) à classer dans l'échelle de mesure de la gêne sensorielle (en bas) selon les difficultés rencontrées en musée.
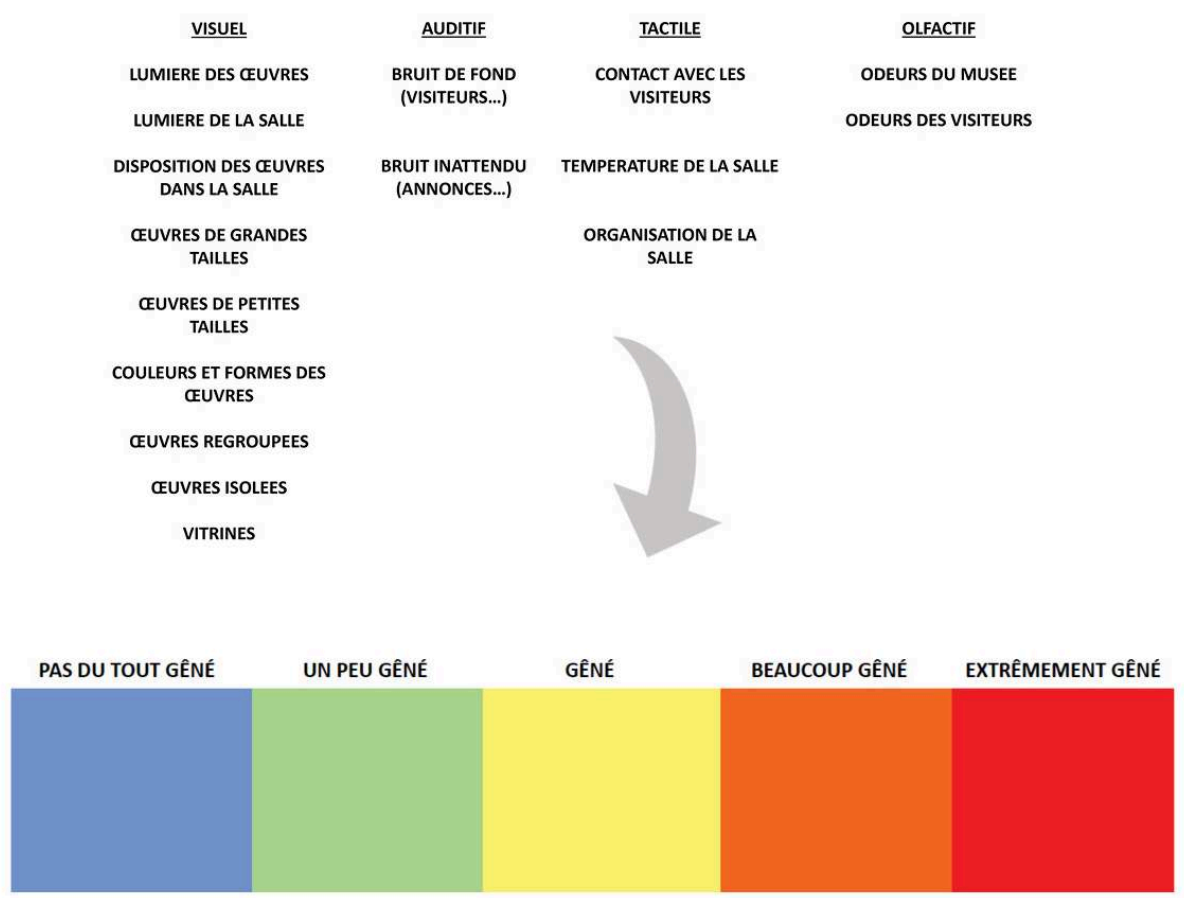

(C) Cyrielle Leriche

\section{Construction de la visite :choix du lieu, des œuvres et préparation du discours}

Une expérimentation sur le terrain a été menée sous la forme de vingt visites individuelles de 45 minutes avec une personne autiste et son accompagnant dans les collections grecques préclassiques du musée du Louvre. Ces visites leur étaient présentées une semaine à l'avance au travers d'un document récapitulant les informations pratiques et le déroulé du programme afin de laisser moins de place à l'imprévu. Le format individuel de la visite a été choisi pour plusieurs raisons : établir une relation privilégiée et intimiste avec chaque sujet, répondre à chaque besoin, pallier les difficultés sociales des personnes avec autisme (un format groupe aurait engendré des variables indésirables et indépendantes de l'expérience, liées au contact du groupe).

Le Louvre est un grand musée avec de nombreux visiteurs et un environnement sensoriel exacerbé. La difficulté sensorielle que représente ce musée pour des personnes avec TSA a constitué pour cette étude un lieu tout à fait propice à la recherche. Le choix unique de la salle des antiquités grecques préclassiques a été 
motivé par son taux de fréquentation modéré et sa localisation géographique au sein du musée (proximité d'une sortie en cas de crise ou de panique et attenante à une salle généralement vide pour avoir un lieu d'apaisement si besoin).

Les œuvres présentées ont également fait l'objet d'une sélection précise : il était impératif de présenter un maximum de six œuvres durant la visite. Les œuvres ont été choisies de façon à avoir un échantillon très hétérogène à présenter aux visiteurs : petites, grandes, en argile, en pierre, en métal, avec vitrine ou sans, isolées ou regroupées...

Enfin, pour ce qui est du discours, le discours scientifique associé à chaque œuvre a été adapté à un public parfois néophyte mais également à un public adolescent comme adulte. Ainsi, chaque œuvre était resituée dans son contexte géographique et chronologique (il est très important de replacer les données spatio-temporelles car les personnes avec TSA peuvent avoir de grandes difficultés à se situer dans le temps). Le discours a toujours été interactif car l'implication active permet d'avoir l'attention du visiteur avec plus de facilité. Pour appuyer ce discours, les supports visuels étaient indispensables au bon déroulement de la visite (cartes, frises chronologiques, reproductions des œuvres, détails...) : avec la pensée en image il fallait représenter tout ce qu'ils ne pouvaient pas voir.

Frise chronologique de mise en contexte pour faciliter la visite.

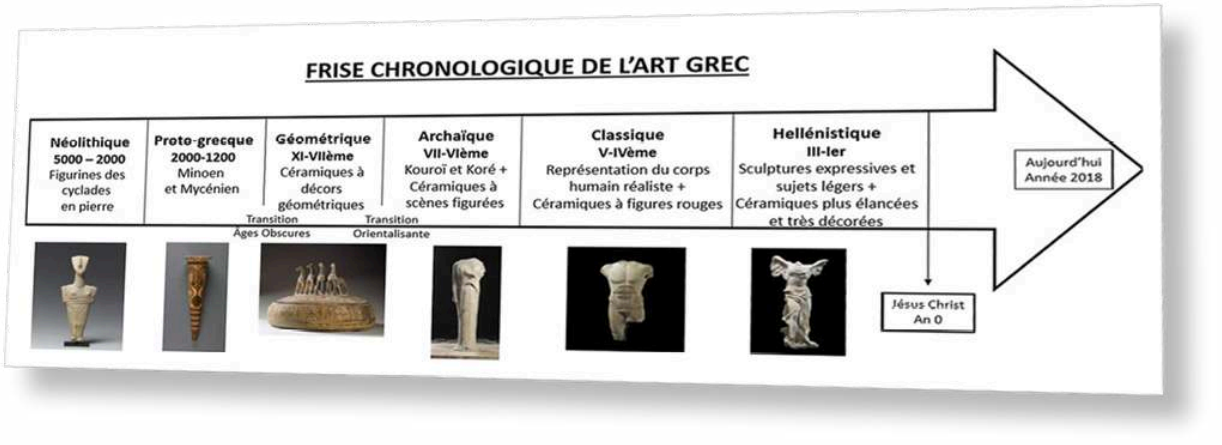

(c) Cyrielle Leriche

\section{Résultats et interprétations de la recherche}

Les résultats ont permis de montrer que la sensorialité n'avait pas forcément d'impact sur la compréhension du discours chez les adultes comme chez les adolescents. La question de la divergence des réceptions sensorielles selon l'âge des participants n'est, d'ailleurs, pas tranchée bien que les adolescents aient rencontré légèrement plus de difficultés à gérer la sensorialité en musée.

L'hypothèse formulée serait que l'adaptation de la visite au travers du temps de visite, $\mathrm{du}$ nombre d'œuvres vues et des supports visuels ont pu amoindrir les troubles sensoriels des sujets en les compensant. Cette hypothèse peut être validée au regard d'une étude menée en 2017 concluant que les programmes spécialisés avec les modifications appropriées améliorent la qualité et la durée de la visite en musée pour des visiteurs autistes avec des troubles sensoriels ${ }^{10}$. 
deuxième hypothèse pourrait être que la sensorialité en musée ne rejoindrait pas une question de difficulté de compréhension mais plus de confort de visite. La question de compréhension serait alors plutôt liée à ce que l'on nomme les " troubles cliniques » qui se sont révélés importants dans la compréhension du discours (comme avec la pensée en détails et les repères spatiaux-temporels). Il se pourrait donc que les troubles cliniques aient joué sur la compréhension (avec les troubles langagiers), ou encore sur la concentration (avec la rigidité et les troubles de l'attention) et que ces problématiques soient plus impliquées dans la compréhension du discours que la sensorialité.

Enfin, les analyses de ces questionnaires ont également mis en avant que les éléments sensoriels les plus problématiques en musée sont liés aux bruits inattendus ou aux bruits de fond et aux contacts avec les visiteurs. Il est important de prendre en compte que ces deux éléments sensoriels sont également ceux qui ressortent le plus du profil sensoriel de Dunn : les troubles du quotidien sont donc identiques à ceux identifiés dans les musées.

Fiche visuelleexplicative de préparationà la visite.
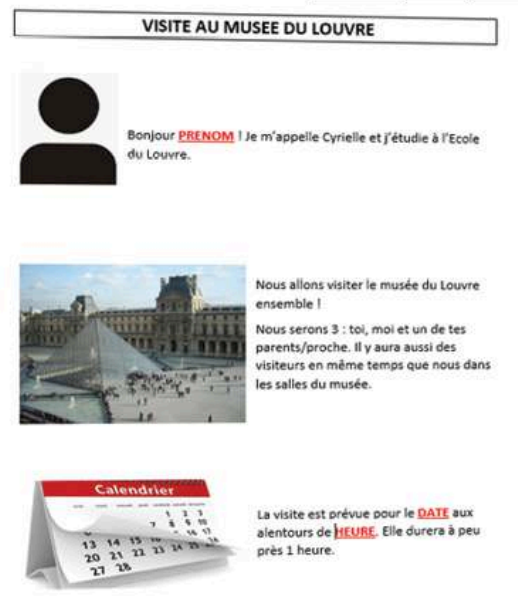

La visite est ortive dour le DATE aux
alentours de hiEURE, Elle durera a peu alentours de
press 1 heure.

Avant de venir, tu peux te renseigner sur le muste du Lowver : C'est un grand muste avec des visiteurs set des annonces audios dons pleins de
differentes qui disent de faire attention a ne pas perdre ses saccs.
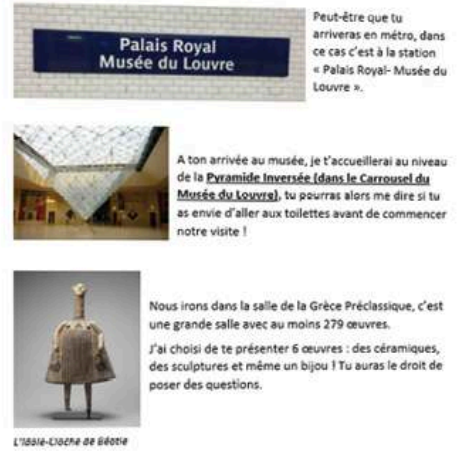

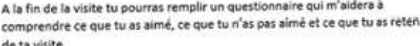
de ta visite. Aores so viste, situ en as envie, tu po
solle, ou meme dans of sutres alles A tres vite ou musie ! cyrielle.

\section{Une remédiation utile des musées et des médiateurs}

Au regard des résultats de cette recherche, des propositions de remédiation ont été avancées pour permettre aux musées de véritablement prendre en charge les personnes présentant des troubles du spectre autistique.

Du côté de l'institution muséale, il semble primordial que les musées indiquent les horaires et jours d'ouverture ayant une affluence moindre sur leurs sites Web afin que des visites spontanées soient rendues possibles. Pour ce faire, il serait bon qu'ils possèdent ou se créent une entrée dédiée, sans queue ni turbulence : une attente trop longue, statique, dans une foule noyée par les impulsions sonores et les contacts physiques non souhaités étant des invitations à rebrousser chemin. Pour favoriser ces 
venues spontanées, les musées pourraient mettre à disposition des visiteurs, via leurs sites Web, des fiches adaptées comme celle du MET ou du musée du Louvre : la préparation en amont étant bien souvent déterminante pour une telle expérience.

Il serait également bienvenu que les musées instaurent des temps calmes avec un accès réduit à l'institution, une luminosité moindre et une absence de fond sonore comme au Children's Museum de New Hampshire ; des moments qui leurs permettraient de se familiariser avec le musée et peut-être, d'y revenir à leur gré.

31 Enfin, certains outils, comme les sacs sensoriels du Victoria \& Albert Museum, semblent être des compléments ingénieux car outre offrir une activité ludique, ils rendent la visite supportable et vivable en mettant en place des aides sensorielles qui permettent aux personnes avec TSA de mieux vivre leurs expériences muséales.

Il est important que les médiateurs reçoivent une réelle formation sur les troubles $d u$ spectre autistique, non seulement sur la façon de les accompagner au musée, mais également sur le trouble en lui-même. Un médiateur informé du trouble saura mieux prendre en charge le visiteur en cas de mal-être, en identifier les raisons, qu'un médiateur uniquement formé à l'accompagner en salle.

Il faudrait que les musées conservent le plus possible le format individuel ou, au mieux, des groupes restreints à quatre ou six personnes au maximum : les troubles sociaux engendrant des angoisses, être intégré à un groupe constitué d'étrangers ne rendrait la visite que plus éprouvante.

L'interaction est également primordiale, puisqu'en échangeant avec les visiteurs, on s'assure qu'ils suivent toujours la discussion malgré leurs troubles de l'attention.

Enfin, il est très important de multiplier les aides à la visite (visuelles notamment) dans les salles ou à la demande - qui permettraient de diminuer leurs difficultés : ces supports facilitant la compréhension, la concentration ou encore la visualisation.

\section{Conclusion}

Les institutions muséales sont des lieux privilégiés pour les personnes présentant des troubles du spectre autistique : elles constituent un fabuleux lieu d'apprentissage social comme culturel.

Il est important que l'autisme soit davantage reconnu, accepté et intégré par la société et il appartient aux musées d'y participer. En ouvrant leurs portes aux personnes avec TSA, les musées pourraient devenir des lieux accessibles, ouverts, visionnaires mais aussi des lieux d'apprentissage des scénarios sociaux pour ces publics.

Toutefois, il est capital de souligner que cette démarche n'est possible que si le musée s'adapte aux troubles du spectre autistique. Prendre en charge l'autisme ne signifie pas créer une visite "type » en partant de ce qu'il se fait ailleurs, mais véritablement construire un projet culturel en rassemblant les connaissances du handicap et les bases du musée pour réaliser un programme où les deux éléments se complètent et se construisent l'un dans l'autre.

Un travail conséquent attend les institutions muséales sur ce chantier, mais celles qui se pencheront sur la question pourraient aussi être des exemples de stratégies et de méthodes novatrices face aux troubles du spectre autistique. Cela contribuerait à 
faciliter la prise en charge de ces publics dans les institutions culturelles et à améliorer de manière plus générale l'accessibilité dans les musées français.

\section{BIBLIOGRAPHIE}

American Psychiatric Association, DSM-5, Manuel diagnostique et statistique des troubles mentaux, Paris : Elsevier Masson, 2013, 947 p.

Caucal, D. et Brunod, R. Les aspects sensoriels et moteurs de l'autisme, Grasse : AFD édition, 2010, 216

p.

Dunn, W. Profil Sensoriel, Paris : CPA, Éditions du centre de psychologie appliquée, 2010, 109 p.

Frith, U. L'énigme de l'autisme, Paris : Édition Odile Jacob, 2010, 323 p.

Grandin, T. Ma vie d'autiste, Paris : Édition Odile Jacob, 1986, 256 p.

Grandin, T. Penser en image et autres témoignages sur l'autisme, Paris : Édition Odile Jacob, 1997, 261

p.

Grandin, T. et Panek, R. Dans le cerveau des autistes, Paris : Édition Odile Jacob, 2014, 190 p.

Treese-Daquin, C. Autisme et Culture : Balades au Musée du Louvre, Grasse : AFD édition, 2016, 157 p.

Dossiers d'œuvres du musée du Louvre : nA276 (Rhyton au poulpe) ; nBJ2169 (Pendentif rhodien) ; $n^{\circ}$ E874 (Dinos du peintre de la Gorgone) ; ${ }^{\circ}$ Ma686 (Koré de Samos) ; nF204 (Amphore bilingue des peintres d'Andokidès et de Lysippidès) ; $n^{\circ}$ Ma2792 (Torse de Milet)

\section{NOTES}

1. Loi $n^{\circ} 2005-102$ du 11 février 2005 pour l'égalité des droits et des chances, la participation et la citoyenneté des personnes handicapées, art. 47.

2. Personne n'ayant pas de troubles du spectre autistique.

3. Cette recherche a été réalisée au musée du Louvre grâce à un droit de parole, mais est totalement indépendante et n'appartient à aucune institution.

4. Leriche, C. Musée et troubles du spectre autistique, Mémoire de master 2 en Muséologie, sous la direction de Marie-Clarté O’Neill, Paris, École du Louvre, 2018, 137 p.

5. Termes définis par l'échelle de Wechsler.

6. Les pistes de visite du musée du Louvre : http://www.louvre.fr/pistes-de-visite Ressources pour les visiteurs avec TSA du Metropolitan Museum of Art : http:// www.metmuseum.org/events/programs/access/visitors-with-developmental-and-learningdisabilities/for-visitors-with-autism-spectrum-disorders

7. Ressources d'aide à la visite pour le jeune public avec TSA du V\&A Museum of Childhood : http://www.vam.ac.uk/moc/learning/sen/visiting-autistic-child

8. Programme pour public autiste du Children's Museum of New Hampshire : http:// www.childrens-museum.org/things-to-do/programs/autism 
9. Programme d'Autisme sans limites pour le public autiste, en partenariat avec le MBAM : http://autismesanslimites.org/partenariat-musee-des-beaux-arts-de-montreal-autisme-sanslimites

10. Silverman, F. et Carr Tyska A. Supporting participation for children with sensory processing needs and their families: community-based action research. American Journal of Occupational Therapy, vol. 71, $\mathrm{n}^{\circ}$ 3, mai 2017, 157 p.

\section{RÉSUMÉS}

Les publics présentant des troubles du spectre autistique nécessitent une prise en charge particulière. Comme le montrent les exemples présentés ici et une étude expérimentale menée par une jeune professionnelle au musée du Louvre, les musées adaptent progressivement leurs outils de médiation. Ils tentent ainsi de répondre au mieux aux troubles cliniques et sensoriels qui impactent la compréhension du discours muséal et le confort de visite.

\section{INDEX}

Mots-clés : Médiation, public handicapé, musée d'art

\section{AUTEUR}

\section{CYRIELLE LERICHE}

Diplômée de muséologie à l'École du Louvre

cyrielleleriche@hotmail.fr 